\title{
An Advanced Platform for Thermodynamics Education. Part two: Monomer Quantum Volume in Pure Fluids
}

\author{
B.I. Sedunov \\ Russian New University (ROSNOU) 22 Radio st., Moscow, Russia \\ E-mail: sedunov.b@gmail.com
}

Received 7 August 2021, Revised 2 October 2021, Accepted 13 October 2021

\begin{abstract}
The paper presents a remarkable application of the advanced thermodynamics education platform to the molar Gibbs energy $G$ for basic particles in neat fluids. From the $G$, named also as the chemical potential, and the monomer fraction density $D_{m}$ functions the Monomer Quantum Volume $V_{q}(T)$ may be computed. The $V_{q}(T)$ variable reflects the quantum uncertainty of basic particles positions in atomic and molecular fluids. It has proven to be universal for all fluid's densities at a fixed temperature $T$. An extraordinary precision of modern thermophysical databases, such as the NIST Webbook, permits an estimation of the Monomer Quantum Volume values millions times lower than the atom's volume! By studying the $V_{q}(T)$ function the students can estimate the quantum uncertainty effects in pure fluids for a total range of their existence up to thousands of Kelvin! The enthalpy data normalization has proven to be very efficient. The advanced platform is highly useful and informative for thermodynamics education. For students it is very educative to study and utilize the author's computer aided big thermophysical data analysis method to form their vision of the atomic and molecular quantum states distribution in pure fluids.
\end{abstract}

Keywords: Pure fluid; thermophysical properties; chemical potential; entropy; quantum effects.

\section{Introduction}

With the pure fluids based educational platform [1] we will here study the quantum effects in the thermodynamics of these fluids utilizing the molar Gibbs energy $G$, named also as the chemical potential for their basic particles.

Pure fluids present an advanced platform for thermodynamics education and research [1] and provide the next step from the widely used simplified classical fluid model, which ignores the quantum uncertainties, being essential factors for the fluids. The quantum uncertainty volume is larger than the own particles' volume in the quantum fluid and smaller than the own particles' volume in others atomic and molecular fluids.

Only the Helium II is considered now as the quantum fluid for its superfluidity [2] or quantum turbulence [3] effects near the absolute zero temperature. But it does not mean that all other pure fluids are free from quantum effects at higher temperatures. They demonstrate the quantum uncertainty effect for basic particles' positions, based on the Heisenberg uncertainty principle [4], in wide ranges of the fluid density and temperature.

\subsection{The useful terms and variables for pure fluids}

The pure fluids' quantum uncertainty features cognition is based on the next terms and variables [1]:

Monomers - the basic particles, temporarily no interacting with other particles.

The monomer fraction density $D_{m}$ - the molar density of the monomer fraction [6];

Basic particle is a particle of a pure fluid, corresponding to its chemical nature. The basic particles molar density $D$ is shown in thermophysical databases;

Free moving particles - monomers and clusters;
The free moving particles molar density $D_{p}=P / R T$;

The Chemical Potential $G$ for all basic particles of a pure fluid being the same as for Monomers [6].

The atomic quantum uncertainty volume $V_{q}$ was introduced by Kittel [7] as the de Broglie's matter wave idea [8] further development for atomic ideal gases.

\subsection{The experimental background of the research}

For the fluids nature cognition and comprehension the computer aided analysis of precise experimental thermophysical data has proven to be extremely informative [9]. The computer aided analysis of big precise experimental thermophysical data for pure fluids may provide for students a clear vision and comprehension of quantum uncertainty effects in all ranges of fluids' temperature and density.

The monomer fraction in pure fluids concept $[5,6]$ opened the way to a chemical potential expression via the monomer fraction density $D_{m}$ and thus to the quantum uncertainty volume $V_{q}$ [7]. A high precision of the NIST Webbook [10] data, given with 12 digits, provides a satisfactory basis for hidden quantum uncertainty effects cognition in many pure fluids. But for it the NIST Webbook data need to be normalized to zero enthalpy values at the absolute zero temperature.

The goal of the paper is to demonstrate and explain the unknown before quantum uncertainty effects in pure atomic and molecular fluids. We strive to estimate the quantum uncertainty volumes for atomic and molecular fluids in the total ranges of their existence. 


\section{The Monomer Fraction Density, $D_{m}$}

The pressure dependent part of the Gibbs energy $G$ for an atomic real gas is the translational part $G_{t r}$ being the same as $G_{t r m}$ for an atomic ideal gas of Monomers:

$G_{t r}=G_{t r m}=R T \ln \left(D_{m} V_{q}\right)$,

where $V_{q}=h^{3} N_{A}^{4} /(2 \pi M R T)^{3 / 2}$ - the atomic molar quantum volume [7], which is proportional to the third degree of the thermal de Broglie wavelength [8]; $h$ is the Plank's constant, $M$ is the basic particles' molecular mass in $\mathrm{kg} / \mathrm{mol}$, $N_{A}$ is the Avogadro number, $R$ is the gas constant. So, $G_{t r}$ has a quantum nature.

For the molar Gibbs energy $\mathrm{G}$ we have the well-known thermodynamics law:

$$
\partial G /\left.\partial P\right|_{T}=V=1 / D
$$

Differentiating the $G_{t r}$ from the Eq. (1) we come to the differential equation for $D_{m}[5,6]$ :

$\partial D_{m} /\left.\partial P\right|_{T}=D_{m} /(R T D)$.

The Eq. (2) reminds the well-known equation for fugacity $f$ introduced in thermodynamics by G. N. Lewis [11]:

$\partial \ln (f) /\left.\partial P\right|_{T}=1 /(R T D)$

The monomer fraction density $D_{m}$ seems to be much more understandable for students than the fugacity $f$, which can be defined as the Monomer fraction partial pressure $P_{m}$ [1].

The Eq. (2) can be solved numerically very precisely for small enough initial $P_{1}$ and the pressure step values $\Delta P_{i}=$ $P_{i}-P_{(i-1)}$. The next form provides a very precise numerical solution [12] for the Eq. (2):

$D_{m i}=D_{m(i-1)}\left(1+\Delta D_{P i} /\left(2 D_{(i-1)}\right)\right) /\left(1-\Delta D_{P i} /\left(2 D_{i}\right)\right)$.

As an initial condition for an extra diluted gas we have: $D_{m l}=2 D_{P l}-D_{l}$. After $D_{m i}$ computation we add to the thermophysical properties table the $D_{m i}$ column, which may serve as an argument for series expansions of other thermophysical functions.

The vague boundary between the monomers' free movement and their bound states in clusters makes difficult the monomer fraction density visualization. But the phenomenological approach via Eq. (2) permits to define correctly the monomer fraction density in fluids.

The difference between densities $D, D_{p}$ and $D_{m}$, is clearly seen from the Figure 1, which shows that in the Nitrogen at a temperature $120 \mathrm{~K}$ and at pressures higher than 10 bar all three densities differ very much.

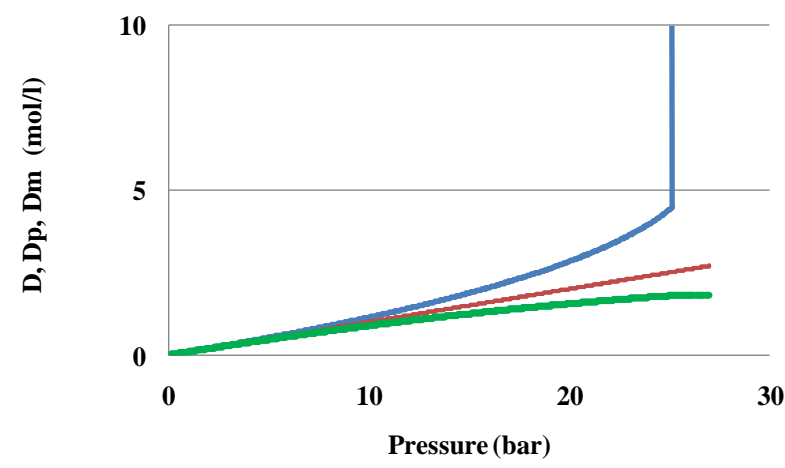

Figure 1. Densities: $D, D_{p}$ and $D_{m}$ for Nitrogen at a temperature $T=120 \mathrm{~K}: D$-blue, $D_{p}-$ red and $D_{m}-$ green lines.

At the vapor saturation, $P_{\text {sat }}=25.1$ bar, $D$ jumps to the liquid state density, $D_{\text {liq }}=18.68 \mathrm{~mol} / \mathrm{l}$, while $D_{p}, D_{m}$ and also $G$ functions stay continuous.

\subsection{The entropy of pure molecular fluids}

The pure molecular real gas entropy $S$ may be split on:

- The pressure independent part for internal molecular vibrations and rotations, $S_{\text {int }}(T)$;

- $\quad$ The pressure dependent part for molecular and clusters' translational movements entropy,

$S_{t r}(T, P)=S(T, P)-S_{\text {int }}(T)$.

From the Eq. (1) we come to the pressure dependent part of entropy $S_{t r}(T, P)[5]$ :

$S_{t r}=H_{t r} / T-R \ln \left(D_{m} V_{q}\right)$.

This equation is the generalization to real gases and molecular fluids of the well-known Sackur-Tetrode quantum equation for an atomic ideal gas [13-15]:

$S=2.5 R+R \ln \left(V / V_{q}\right)$.

The students may test the Eq. (5) for different pure fluids with known precise thermophysical properties.

\subsection{The quantum volume density dependence}

First, we compute $G=H-T S$, then $D_{m}$ and $\ln \left(V_{q}\right)$. The Eq. (1) may be reduced to a form:

$\ln \left(V_{q}\right)=G / R T-\ln \left(D_{m}\right)$.

For all investigated pure fluids the computed isothermal values for $\ln \left(V_{q}\right)$ have proven to be stable in wide ranges of densities.

For atomic real gas Helium at $200 \mathrm{~K}$ the $\ln \left(V_{q}\right)$ stability is better than $10^{-5}$, Figure 2 . 


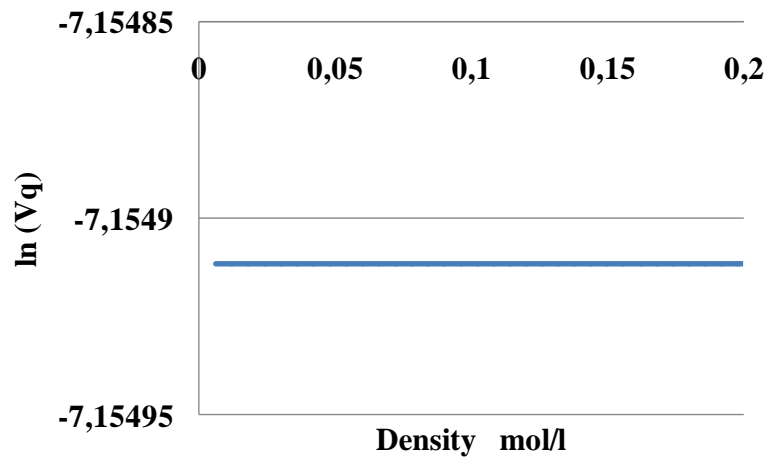

Figure 2. The $\ln \left(V_{q}\right)$ stability for Helium at $200 \mathrm{~K}$ at a level better than $10^{-5}$.

This stability may be explained by the quantum uncertainty of individual particles positions, independent on their entrance in clusters or in the liquid phase. This high stability is also confirmed by the case of the $\ln \left(V_{q}\right)$ for molecular gas Parahydrogen at 72 K, Figure 3.

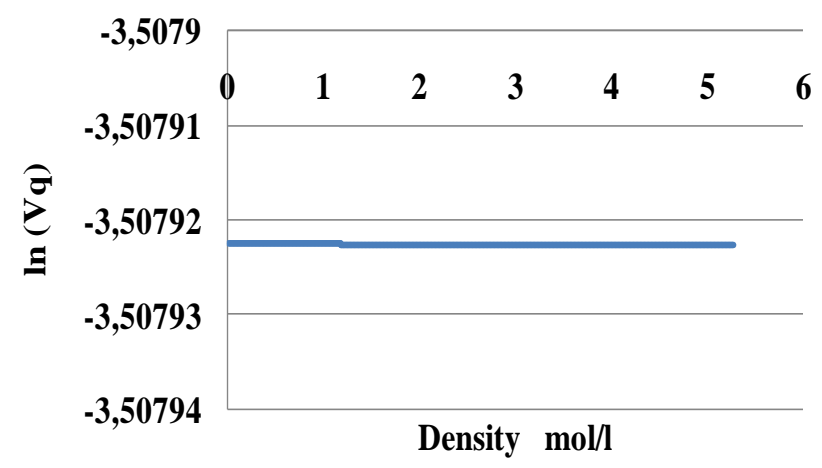

Figure 3. A remarkable high stability of the $\ln \left(V_{q}\right)$ in Parahydrogen at $72 \mathrm{~K}$.

In spite of a small scale at Figures 2, 3 the deviations of $\ln \left(V_{q}\right)$ from a constant are not seen. To estimate more carefully the deviations level we take the difference between the $\ln \left(V_{q}\right)$ and its average value. This difference may be zoomed: what means to be presented on a diagram in a more corresponding scale, Figure 4.

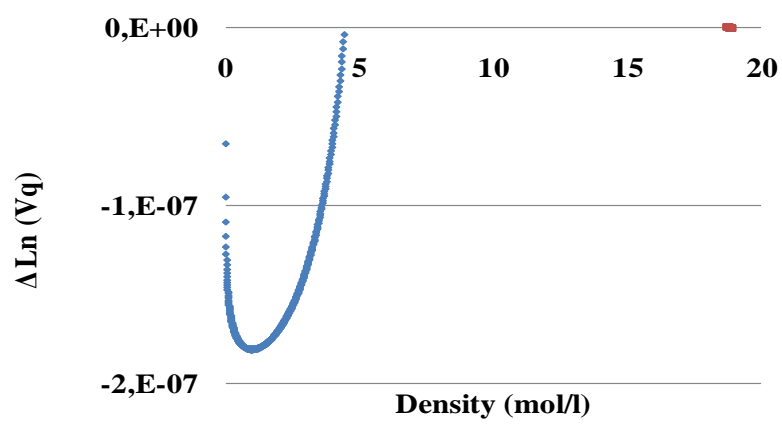

Figure 4. Deviations of $\ln \left(V_{q}\right)$ in Nitrogen from an average value $\ln \left(V_{\text {qav }}\right)=-14.07$ at a temperature $T=120 \mathrm{~K}$ : The vapor phase - blue markers; The liquid phase - red markers.

The deviations seen at the Figure 4 seem to origine not from the physical, but from the mathematical nature, caused by errors at the $D_{m}$ computation. To test this idea we redownloaded the data table with a doubled pressure step value and estimated new deviations, Figure 5.

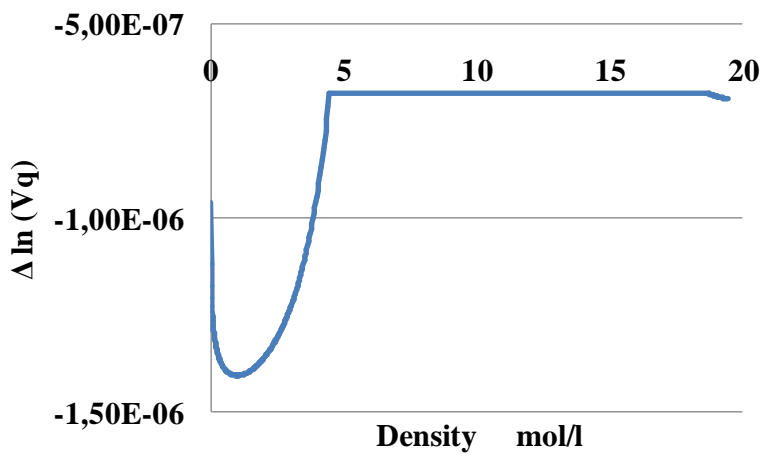

Figure 5. Deviations of $\ln \left(V_{q}\right)$ in Nitrogen from an average value $\ln \left(V_{\text {qav }}\right)=-14.07$ at a temperature $T=120 \mathrm{~K}$ for $a$ doubled pressure step value.

We see that the pressure step value doubling resulted in the 5-fold deviations growth! At the step diminishing we could have smaller deviations. So, the apparent deviations have the mathematical nature. We conclude that the quantum uncertainty volume really does not depend on the fluid density in spite of visible deviations.

\section{The quantum volume temperature dependence}

The quantum uncertainty volume is a unidimentional function; it does not depend on the fluid density and depends only on temperature. To study the temperature dependence we should process either many isothermal data tables or one isochoric table. But the isochoric table has a limited temperature range. So, to cover the whole range for each fluid we have to create around 10 data tables for different temperatures.

\subsection{The quantum volume isochoric analysis}

The isochoric $\ln \left(V_{q}\right)$ dependence on the $\ln \left(T / T_{1}\right)$ for Nitrogen real gas is shown at the Figure 6.

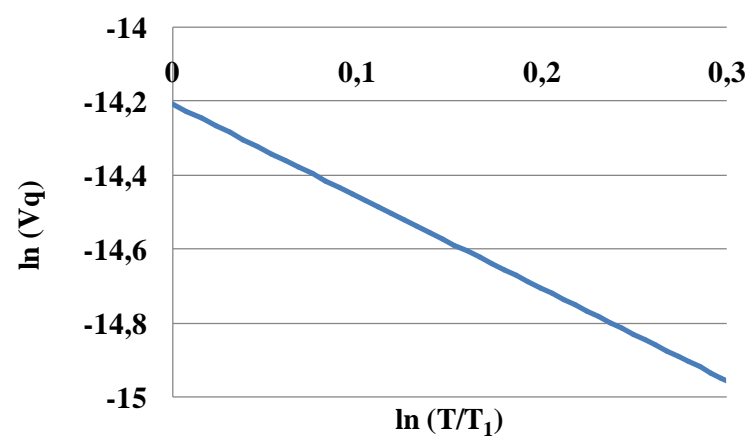

Figure 6. The isochoric $\ln \left(V_{q}\right)$ dependence on $\ln \left(T / T_{1}\right)$ for Nitrogen real gas in the 127 - $180 \mathrm{~K}$ temperature range with volume $303 \mathrm{l} / \mathrm{mol}$ and $T_{1}=127 \mathrm{~K}$.

We have downloaded from the NIST Webbook [10] the isochoric data for the Nitrogen real gas with molar volume $303 \mathrm{l} / \mathrm{mol}$ and the $127-180 \mathrm{~K}$ temperature range. We have selected such a large volume to use for $D_{m}$ a simple expression: $D_{m}=2 D_{p}-D$. 
The logarithm of the quantum uncertainty volume linearly depends on the $\ln \left(T / T_{1}\right)$ with a tangent equal to 2.495. This number almost equals to $2.5=i / 2$, the half of the molecular degrees of freedom number $i$. The larger is the molecular degrees of freedom number $i$, the quicker quantum uncertainty volume falls with temperature.

The molecular quantum uncertainty volume falls with temperature as $\left(T_{I} / T\right)^{i / 2}$, Figure 7 . This fall at $2000 \mathrm{~K}$ reaches $10^{-8}$ from the liquid Nitrogen molar volume, $V_{\text {liq. }}$. The high precision of the NIST Webbook data provides the quantum effects estimation at these extreme conditions!

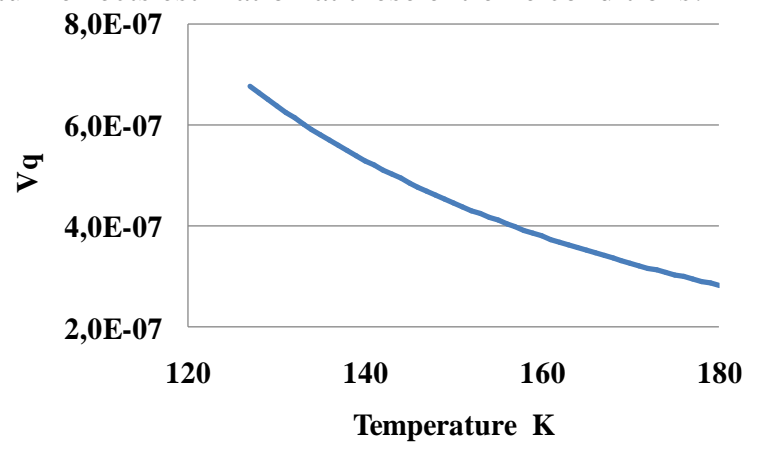

Figure 7. The molecular quantum uncertainty volume in Nitrogen falling with temperature as $\left(T_{I} / T\right)^{i / 2}$.

The $V_{q}$ to $V_{\text {liq }}$ ratio is very small: around $5 \mathrm{E}-6$ at $180 \mathrm{~K}$.

\subsection{The quantum volume isothermal analysis}

By a number of isothermal data tables, we expand the analysis range to all temperatures presenting the fluid in the database. For Nitrogen this range is from 63.2 to $2000 \mathrm{~K}$. We have built the diagram of the quantum volume logarithm dependence on the $\ln \left(T / T_{1}\right)$ with $T_{1}=63.2 \mathrm{~K}$, Figure 8 .

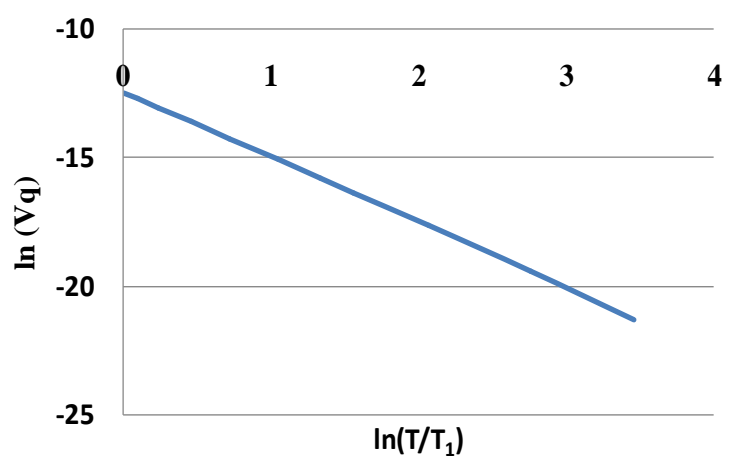

Figure 8. The $\ln \left(V_{q}\right)$ values dependence on the $\ln \left(T / T_{1}\right)$ with $T_{1}=63.2 \mathrm{~K}$, for Nitrogen in the range from 63.2 to $2000 \mathrm{~K}$.

In spite of an extra wide temperature range, the graph is linear, with a tangent equal to -2.494 . We see that this tangent is almost the same as for isochoric case. For two atomic molecular gas $\mathrm{N}_{2}$ the tangent is close to $5 / 2$, what differs from the Kittel's 3/2 for atomic gases [7].
The Figure 9 demonstrates the difference between atomic (Argon) and molecular (Nitrogen) gases.

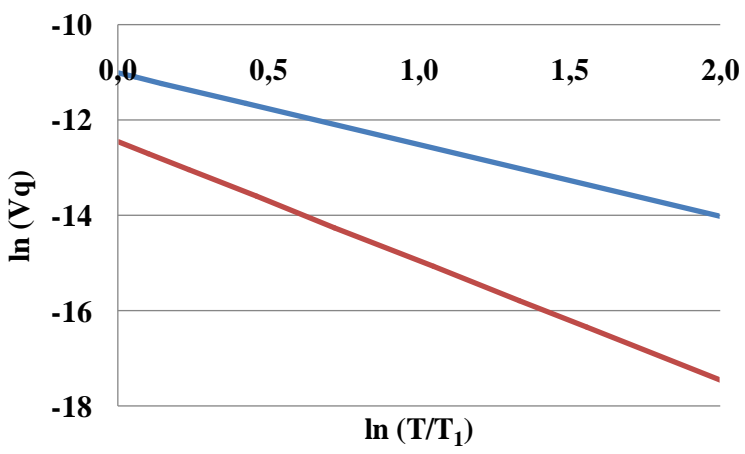

Figure 9. The $\ln \left(V_{m q}\right)$ dependences on the $\ln \left(T / T_{1}\right)$ for Argon (blue line) and Nitrogen (red line).

Different tangents reflect different degrees of freedom numbers, but not for all fluids.

One more molecular gas, Oxygen, happened to have the $\ln \left(V_{m q}\right)$ linear dependence on the $\ln \left(T / T_{1}\right)$, Figure 10.

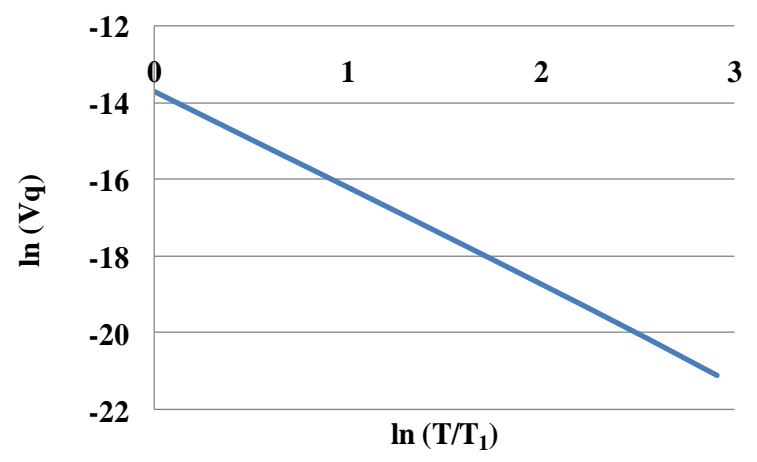

Figure 10. The $\ln \left(V_{q}\right)$ values dependence on the $\ln \left(T / T_{1}\right)$ with $T_{1}=54.3 \mathrm{~K}$, for Oxygen in the range from 54.3 to $1000 \mathrm{~K}$.

Not all fluids show this linearity because of irregular conventions for fluids' Standard states in the NIST Webbook [10]. The problem of the tangent correspondence to the molecular degrees of freedom number is waiting for more regular databases, with a universal convention for Standard states.

We will try to correct the initial enthalpy data for some fluids to escape from the $\ln \left(V_{m q}\right)$ on the $\ln \left(T / T_{1}\right)$ graph curvature.

\subsection{The quantum uncertainty effects in irregular fluids}

The strait lines for $\ln (V q)$ versus $\ln \left(T / T_{1}\right)$ exist only on diagrams for Argon, Oxygen and Nitrogen. For other fluids the graph is curved, like for Helium, Figure 11. 


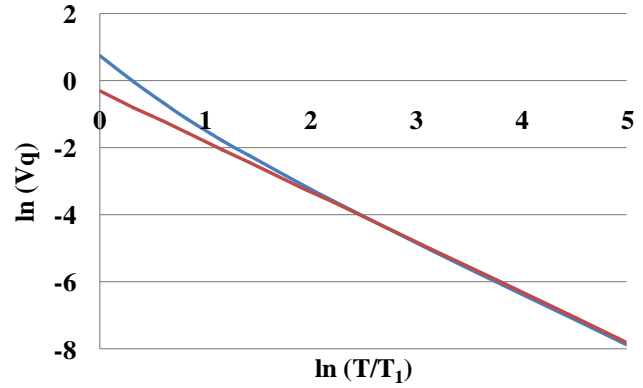

Figure 11. The $\ln \left(V_{q}\right)$ dependence on the $\ln \left(T / T_{1}\right)$ for Helium (blue line) and the high T trend (red line).

We see that the curvature finishes at $\ln \left(T / T_{1}\right)>2$. So, we build the high $T$ trend line for this range with the same tangent as for Argon. The trend's tangent is near 3/2.

A similar picture is seen for molecular gas Parahydrogen, Figure 12, but with the trend's tangent near $5 / 2$.

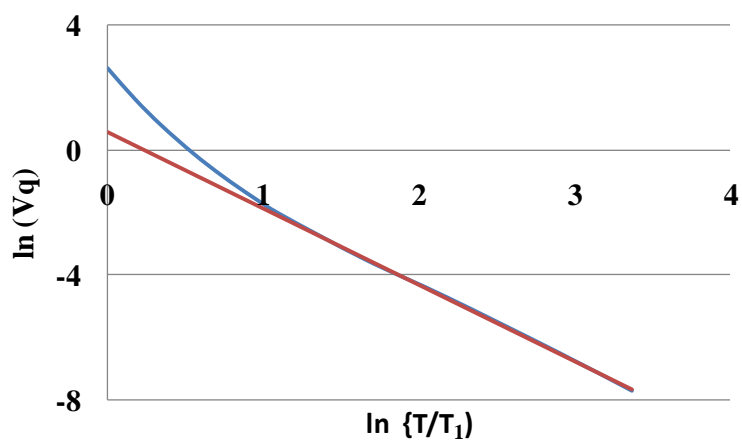

Figure 12. The $\ln \left(V_{q}\right)$ dependence on the $\ln \left(T / T_{1}\right)$ for Parahydrogen (blue line) and the high $T$ trend (red line).

For some fluids we have to analyze the linear high $\mathrm{T}$ trends of their curved graphs.

The Figure 13 demonstrates the $\ln \left(V_{q}\right)$ linear trends dependences on the $\ln (T)$ for atomic gases: Argon and Helium.

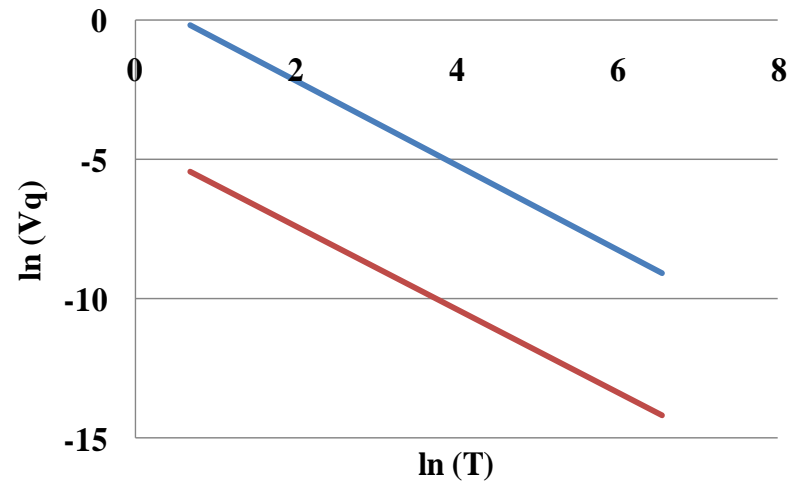

Figure 13. The $\ln \left(V_{q}\right)$ linear trends dependences on the ln (T) for atomic gases: Argon and Helium.

\subsection{The Monomer Quantum Volume correlation for different gases}

The low $T$ curvature may be explained by irregular conventions in the Webbook [10]: only for atomic (Argon) and molecular (Nitrogen and Oxygen) gases both Enthalpy and Entropy data are zero at $0 \mathrm{~K}$, but for some other fluids there are other conventions.

In the online database, such as the NIST Webbook [10], there is no universal convention on the enthalpy zero values. Here we will try to fit the enthalpy data for different fluids to zero values at the absolute zero temperature.

A wonderful linearity of the $\ln \left(V_{m q}\right)$ dependence on the $\ln (T)$ and the trends' lines tangents coincidence with the half of the degrees of freedom numbers are not supported by a similarly clear correlations with atomic $M_{a}$ or molecular $M_{m}$ masses.

The distance between lines at the Figure 13, equal to 5.2 , is much over the $3 / 2 \ln \left(M_{A r} / M_{H e}\right)=3.45$.

So, the Kittel's formula:

$V_{q}=h^{3} N_{A}^{4} /(2 \pi M R T)^{3 / 2}$

does not account for quantum effects in gases in a full scale because of no universal Standard states.

The Figure 14 shows the $\ln \left(V_{q}\right)$ linear trends on $\ln (T)$ dependences for molecular gases: Oxygen, Nitrogen and Parahydrogen.

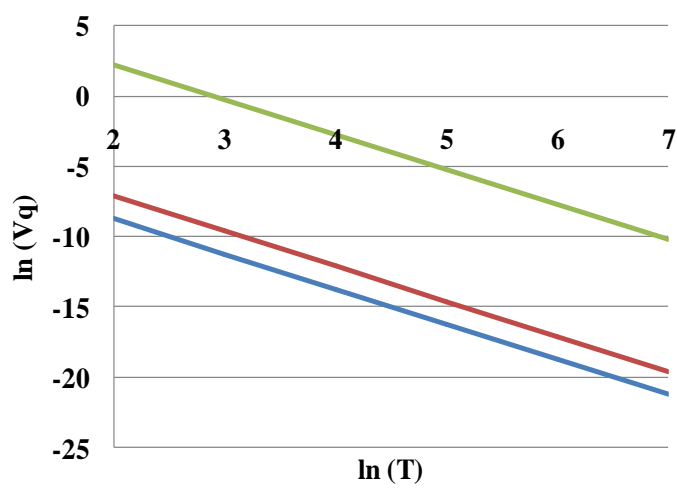

Figure 14. The $\ln \left(V_{q}\right)$ linear trends dependences on $\ln (T)$ for molecular gases: Oxygen - blue, Nitrogen - red and Parahydrogen-green lines.

And again the distances between lines do not reflect precisely the molecular mass difference because of no universal Standard states.

So, the universal convention for Standard states of different fluids is urgently needed!

\subsection{The enthalpy data renormalization}

We have tested the renormalization method on the atomic gas Xenon, for which the initial enthalpy data are not equal zero at the absolute zero temperature, Figure 15. We have renormalized the enthalpy data to be equal zero at the absolute zero temperature by subtraction $1113 \mathrm{~K}$ from the H/R data. The tangent of both lines' slopes has proven to be 2.499 , almost 2.5 . 


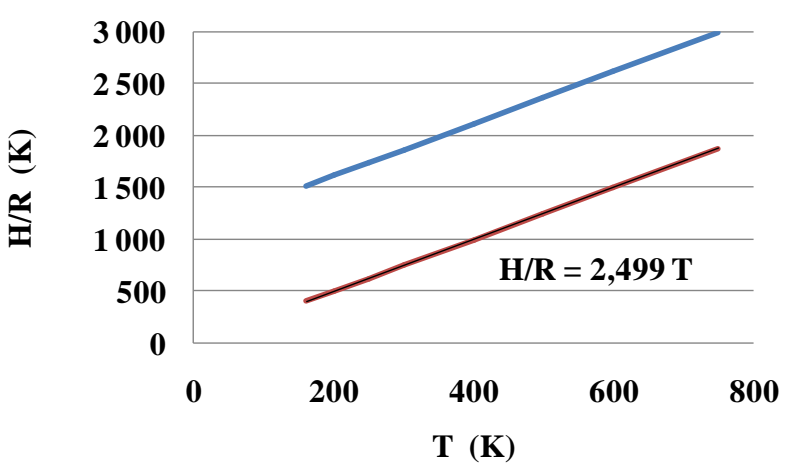

Figure 15. The H/R normalized linear dependence on the Temperature for atomic gas Xenon - red line; Initial dependence - blue line.

We have compared the $\ln \left(V_{q}\right)$ values for initial and normalized data and discovered that the normalized $\ln \left(V_{q}\right)$ graph has become a straight line, Figure 16.

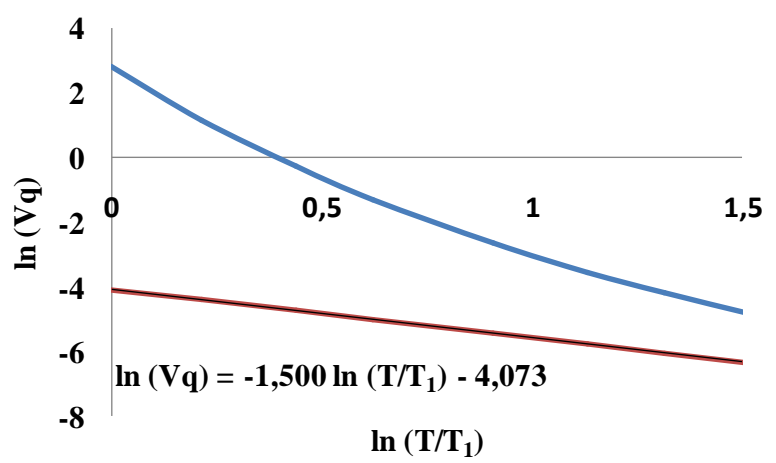

Figure 16. The $\ln \left(V_{q}\right)$ normalized linear dependence on the In $\left(T / T_{1}\right)$ for atomic gas Xenon - red line; Initial curved dependence - blue line.

The straight line tangent of slope has proven to be $-i / 2$ $=-1.5$. The same is true for all Noble gases. It proves the effectiveness of the renormalization method.

\section{The valuable lessons:}

- The contained in online databases, such as the NIST Webbook [10], extra precise experimental data permit to download the isothermal thermophysical properties table for a selected pure fluid and provide a required precision of the $D_{m}$ and $V_{q}$ values computation.

- The key to quantum effects extraction from precise experimental thermophysical data for pure fluids appeared only with the monomer fraction density (MFD) $D_{m}$ introduction in 2007 by the author to the thermodynamics terms and functions family [5]. This idea had been developed further in 2008 in the paper for the International Journal of Thermodynamics [6].

- The Monomer Fraction Density permits the SackurTetrode quantum equation generalization to the molecular fluids' entropy, which provides the Monomer Quantum Volume $V_{q}$ discovery.
- Only after the monomer fraction density natural logarithm, $\ln \left(D_{m}\right)$, from $G / R T$ extraction the strong independence of the quantum volumes for atomic and molecular fluids on the fluid density could appear.

- The computation of the Monomer Quantum Volume $V_{q}$ discovers quantum properties of pure fluids. This value has proven to be universal for all fluid's densities at a fixed temperature $T$.

- The enthalpy data renormalization has proven to be very effective.

\section{Conclusions}

- The unique feature of pure fluids: the uniformity of the basic particles' Chemical Potential for monomers and the total fluid provides the fluid quantum uncertainty volume cognition.

- The Monomer Fraction Density, as a key variable in the Thermodynamics of pure fluids, supports discovering of their quantum uncertainty volume values.

- The found $\ln \left(V_{q}\right)$ values in a wide density range show a strong independence on the fluid density. It tells that the Monomer Quantum Volume is the unidimentional function of temperature $T$.

- The $V_{q}(T)$ variable reflects the quantum uncertainty volume temperature dependences for atomic and molecular fluids.

- The molar Gibbs energy $G$ to the $\ln \left(V_{q}\right)$ linear dependence demonstrates its quantum nature.

- The enthalpy data renormalization in thermophysical databases is strongly recommended.

- The quantum uncertainty phenomena in pure fluids open new direction in the thermodynamics education and stimulate new investigations and discoveries.

\section{Nomenclature}

$D_{m} \quad$ MFD, Monomer Fraction Density, $m o l / l$

$D$ Molar density of basic particles, $\mathrm{mol} / \mathrm{l}$

$\mathrm{V}_{\text {liq }}$ Molar volume of liquid at a triple point, $l / \mathrm{mol}$

$P \quad$ Pressure, bar

$T$ Temperature, $K$

$i \quad$ The number of degrees of freedom

E Molar internal energy, $\mathrm{J} / \mathrm{mol}$

$H \quad$ Molar enthalpy, $J / \mathrm{mol}$

$G \quad$ Molar Gibbs energy (Chemical potential), J/mol

$G_{t r} \quad$ Translational part of the molar Gibbs energy

$G_{\text {int }}$ Intramolecular part of the molar Gibbs energy

$S \quad$ Molar entropy, $J /(\mathrm{mol} \mathrm{K})$

$S_{\text {int }}$ Intramolecular rotations and vibrations entropy

$S_{t r} \quad$ Translational part of the entropy, $J /(\mathrm{mol} \mathrm{K})$

$V_{q} \quad$ Molar quantum volume for basic particles, $l / \mathrm{mol}$

$h \quad$ Plank's constant

$M \quad$ Molecular weight, $\mathrm{kg} / \mathrm{mol}$

$N_{A} \quad$ Avogadro number, $1 / \mathrm{mol}$

$R \quad$ Universal gas constant, $J /(\mathrm{mol} \mathrm{K})$. 


\section{References}

[1] B. Sedunov, An Advanced Platform for Thermodynamics Education. Part one: Small Density Pure Real Gases, Int. J. of Thermo., vol. 23 (3), p.p. 224-232, 2020.

[2] E.M. Lifshitz, J.B. Sykes, Theory of superfluidity of helium II. Reprinted from Uspekhi fizicheskikh nauk 34, Part 4, p. 512, 1948., Ed.: L.P. Pitaevski. Perspectives in Theoretical Physics; Pergamon, p.p. 243-282, 1992.

[3] S. W. Van Sciver, C. F. Barenghi, Ch. 5 Visualisation of Quantum Turbulence, Editors: M. Tsubota, W.P. Halperin. Progress in Low Temperature Physics; Elsevier, vol. 16, p.p. 247303, 2009.

[4] W. Heisenberg. Physics and Philosophy; N.Y. Harper. 1958.

[5] Sedunov B., 2007, Monomolecular fraction in real gases, Proceedings of Joint European Thermodynamics Conference, JETC IX, p.p. 176180.

[6] Sedunov B. Monomer fraction in real gases. Int. J. of Thermo. - 2008. vol. 11(1). - p.p. 1-9.

[7] Kittel, Ch., Thermal physics, 1969, John Wiley and Sons, Inc., New York.

[8] Louis de Broglie. The reinterpretation of wave mechanics. Foundations of physics. vol. 1, 1970, p.p. 5-15 https://doi.org/10701/BF00708650

[9] Sedunov B. Equilibrium molecular interactions in pure gases. J. of Thermodynamics. vol. 2012. Article ID 859047. - 13 pages. DOI: $10.1155 / 2012 / 859047$

[10] NIST Webbook: Thermophysical Properties of Fluid Systems. - 2021.

[11] G.N. Lewis and M. Randall, Thermodynamics and the Free Energy of Chemical Substances, McGraw-Hill Book Company, New York, 1923.

[12] Sedunov B., New approaches to teaching the Thermal Physics of Fluids, ICCT, Tsukuba, Japan, 2010.

[13] Tetrode, H., 1912, Ann. der Physik, 38, p. 434.

[14] Sackur, O., 1913, Ann. der Physik, 40, p. 67.

[15] Grimus W. On the 100th anniversary of the Sackur-Tetrode equation. arXiv:1112.3748v2 [physics.hist-ph] 23 Jan 2013. 